\title{
Magnetron High Power System Design
}

\author{
Alberto Leggieri ${ }^{1,2}$, Davide Passi ${ }^{1}$, Giuseppe Felici ${ }^{2}$, Silvia De Stefano ${ }^{2}$ and Franco Di Paolo ${ }^{1}$ \\ ${ }^{1}$ Università degli Studi di Roma “Tor Vergata”, Dipartimento di Ingegneria Elettronica, Roma, Italy \\ ${ }^{2}$ S.I.T. - Sordina IORT Technologies S.p.A., Aprilia (LT), Italy \\ alberto.leggieri@uniroma2.it
}

\begin{abstract}
This paper describes the design of an X-Band Magnetron to Linear Accelerator (LINAC) High Power Transfer System consisting in an 8 resonant cavities Magnetron connected to a dedicated vacuum Dielectric Window (DW). A main analytical design of devices is shown with compact formulas and is followed by numerical optimization. Electromagnetic and Thermomechanical coupling between these devices is described, particular attention has been given to the Magnetron power coupling and DW matching. A Multiphysics modeling is proposed to consider thermal-structural effects due to the cathode heating for the Magnetron and the Joule effect for the DW. Thermal induced degradation of the device performances are estimated and proper materials and shapes are chosen in order to ensure the desired behavior of the system in operative conditions. The proposed study provided the evaluation of the Magnetron Working Points and DW Scattering parameters in Thermo-mechanical operative conditions. Technological strategies for device coupling are shown.
\end{abstract}

Keywords - Computational Electromagnetics; Dielectric Windows; Electron Linear Accelerators; FEM methods; High Power Microwave Systems; Magnetron; Multi-physics modeling

\section{INTRODUCTION}

Vacuum Tubes (VT) devices are well alive in all applications where huge RF power and intrinsic strong resistance to mismatching are of fundamental importance. Typical field of applications are Meteorological Radar, Microwave Owens and Brazing Furnaces, Particle Accelerators and plasma Physics.

Even if in some other applications, like in the field of wideband airborne and spacecraft telecommunications where size is a major concern, there is an increasing need of a Solid State (SS) technology alternative, SS vs. VT competition makes sense only in some frequency bands and for some power levels.

For example, in the range of millimeter or sub-millimeter waves, and signal power levels greater than kilowatt no SS vs. VT competition exists: here, VT's like Gyrotrons or Free Electron Lasers have the dominance [1-4].

Instead, especially where a narrow spectrum with high RF power is necessary, Magnetrons are the most useful and cheap VT's to be employed.

For example, in LINAC field Magnetrons are the preferred source together with Klystrons [5-7].

Magnetrons generate the High power microwave needed to accelerate particles through exerting the electric force over them. Microwaves are transferred from the source to the LINAC through power transport waveguide (WG), which are typically filled by air or gas.

In order to separate the Ultra High Vacuum (UHV) atmosphere in the tube to the normal atmosphere in the WG, ensuring the microwave power transmission, DW's are employed [8-11]. These windows are made by a waveguide section, in which a dielectric plate is inserted. Both Magnetrons and LINAC's are in UHV, and connected by a
WG; hence two DW's are needed.

Magnetrons are Vacuum Tube oscillators which produces such signals by transferring energy from an electron stream to a RF field. In the proposed study we have employed an eight key-hole cavities X-Band Magnetron operating in $\pi$ mode, with copper anode and tungsten cathode suitable for LINAC applications [12-14].

The High Power Transfer System is composed by the proposed Magnetron and a dedicated DW for which the analytical approach and numerical design are briefly illustrated.

The coupling conditions of Magnetron and DW are analyzed: Both Electromagnetic power coupling of the Magnetron and DW matching are described in this paper. Moreover the thermo-mechanical coupling between these devices is considered.

A Multiphysics modeling is proposed to consider thermalstructural effects due to the cathode heating for the Magnetron and the Joule effect for the DW. In these physically different contests, the thermal coupling and the thermostatation between these devices are considered.

Several studies and different simulation strategies have been performed on the DW [8-11] and Magnetron [12-16] technologies, however we propose to consider the relationships between thermo-mechanical effects and electromagnetic behavior of such devices.

Since the High Power Transfer System is exposed to multiple physic influencing factors, a multiphysics approach has been employed in order to consider the effect of Thermo-mechanical Stresses related to the cathode heating over the EM resonance and the particle motion inside the Magnetron and to consider the Joule Heating and Thermal Expansion (JHTE) effects due to the high powers in transit inside the DW. 


\section{ALBERTO LEGGIERI et al: MAGNETRON HIGH POWER SYSTEM DESIGN}

The thermal contacts with the LINAC, the external environment and the mechanical constraints have been considered in the proposed model.

The connection diagram of a High Power Transfer System form Magnetron to LINAC is represented in Fig. 1. The heat flux is driven to all the components as for the microwave energy.

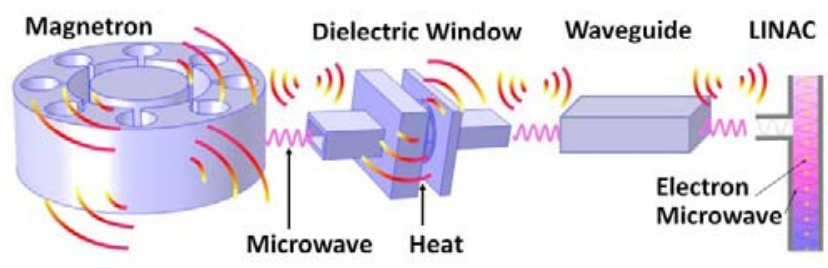

Fig.1. High Power Transfer System form Magnetron to LINAC: Microwave transfer and Heat conduction are schematized.

\section{MAGNETRON DESIGN}

\section{A. Magnetron Operating Principles}

Magnetrons are basically diodes with a cylindrical cathode inserted coaxially in a cylindrical anode, which consists of a set of resonant cavities [12] forming a slow wave structure. Electrons are emitted thermoelectrically by the cathode, and extracted by an opportune anode-cathode voltage.

These electrons are deflected by a static magnetic field oriented in the axial direction, in order to let electrons oscillating between the anode resonant cavities, describing epicycloids. During this process, the electron velocity decrease, and with it, also its energy decreases since is transferred to a RF oscillating field.

Magnetrons belong to the M-type devices, since electrons drift across a magnetic field in crossed electric and magnetic fields [13].

Power is extracted from one resonator, one method being a coupling loop antenna which forms a part of the output circuit. The combination of resonant cavities, end spaces, and output circuit is called the resonant system [12]. The loop antenna consists of a magnetic probe implemented through a single winding coil placed so that the microwave magnetic field can flux through the surface wrapped by the winding.

Since the loop terminates on a Transverse Electric Magnetic (TEM) transmission line and the high power systems are based on Transverse Electric (TE) rectangular waveguide, a transition from coaxial to waveguide, the endlauncher, is need. Radio-frequency power can be also is extracted through a true waveguide output, which terminates in a waveguide coupler [12].

The waveguide output interface can be insulated from the external atmosphere by a dielectric window or by a employing a particular coaxial termination. Magnetron section with power extraction circuit shown in Fig. 2.

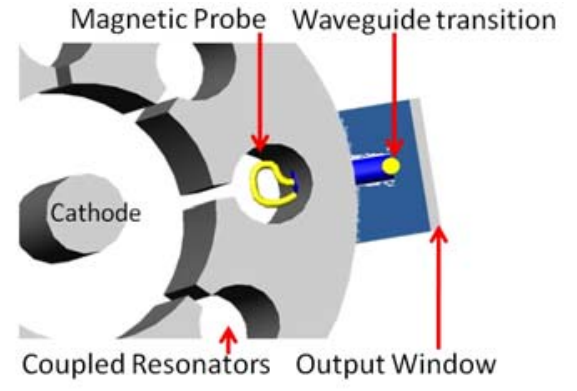

Fig.2. Magnetron section with power extraction circuit

\section{B. Analytical Approach}

The system of coupled cavities can resonate at a specific eigenvalue frequency. One can be chosen for the magnetron operation, basing on the specific application. The resonant mode of the Magnetron is called Normal Mode (NM) and characterized by a specific frequency. The definition of NM identifies the field distributions of coupled oscillators as the phase shift of the fields between two contiguous resonant cavities of the coupled system. By selecting opportune NM frequency, axial static magnetic induction $B$ and anode cathode voltage $V$, electrons can always oscillate and are not captured by the anode. This condition occurs when the static fields equal a particular value called critical field related to the chosen NM.

The critical field is obtained when the anode-cathode voltage equals the critical voltage $V_{c}$ and the axial magnetostatic induction equals the critical induction $B_{c}$. Critical voltage of proposed device, derived by manipulating formulas in [14], is given by (1):

$$
V_{C}=\frac{e}{8 m} B^{2} b^{2}\left(1-\frac{a^{2}}{b^{2}}\right)
$$

with $a$ and $b$ are respectively the cathode and anode radii, $B$ is the Magnetic induction field applied along the axial direction.

Critical magnetic induction, derived by manipulating the linear Magnetron formulas in [14,17], can be described by (2):

$B_{C}=\sqrt{\frac{2 m V}{e d^{2}}}$

where $V$ is voltage applied between anode and cathode and $d$ the anode cathode. Critical values are much higher than the typical operative values, which are chosen in order to maximize the Magnetron efficiency [13,17]. For power generation $V$ needs to be minor of the critical value.

The preliminary analytical design starts by choosing the magnetic induction $B$ which needs to be major than $B_{c}$. 


\section{ALBERTO LEGGIERI et al: MAGNETRON HIGH POWER SYSTEM DESIGN}

The interaction of the particle in the slow wave resonator region is ensured if the cyclotron frequency of the electrons equals the NM resonant frequency $f$ for the desired mode, which will correspond to the frequency of the generated microwave power. This condition yields the relation of $B(3)$ [17]:

$B=\frac{2 \pi f m}{e}$

The voltage $V$ is then fixed ensuring the condition $V<V_{c}$ and the critical magnetic induction is calculated according to the geometry of the resonator, as given by (4) [17]. Afterwards the condition of $B>B_{c}$ is checked.

$B_{C}=\sqrt{\frac{8 V m}{e}} \frac{1}{b\left(1-\frac{a^{2}}{b^{2}}\right)}$

For this device, the design $\pi$ mode frequency is $f=9 \mathrm{GHz}$, then $V_{c}=130 \mathrm{KV}$ with $B_{c}=1359 \mathrm{G}$. Chosen operative values are $V=60 \mathrm{KV}$ and $B=1330 \mathrm{G}$. The analytical calculations shown above simplify the design by providing initial values which must be optimized by an electromagnetic simulation; however the magnetic induction needs to be near the critical value.

\section{Magnetron Multiphysics Aspects}

During the Magnetron operation, the power dissipation of the cathode produces a considerable temperature increase and induces a thermal expansion of both the cathode and the anode. Significant displacements of the resonant structure alter the desired EM behavior.

In order to characterize the coupled cavities resonant system of Magnetrons, an Eigen-frequency (EF) study is needed; this allows to find frequencies relative to all permitted resonant normal modes. By performing an eigenvalue analysis we can estimate these resonant modes, describing their electric field power density distribution in the transversal cross section of the Magnetron. By a Thermal Stress (TS) stationary analysis we can determine temperature and deformation [17-18], when the heat generated by the cathode power dissipation has been diffused on all the reachable Magnetron components and the system has become thermally stable, since the external temperature is by then steady over all the Magnetron outer surfaces, which are exposed to the external environment. Since the Electrostatic (ES) fields are altered by the particles presence, we have a two-way coupling between the particles and field: the field exerts a force on the particles and the particles exert a space charge on the field. Moreover, the variables of the problem are also dependent on the Coulomb forces interactions between the particles.
For these reasons, is necessary to solve the problem for the particles and fields simultaneously by couple a Particle Tracing (PT) and an ES analysis. These coupling effects inside the acceleration-deflection space require a time dependent (TD) analysis, to obtain a characterization of the particle trajectories with a description of energy and velocity. It allows besides to estimate the cathode electron density and the charge distribution of the electron clouds in the transversal cross section of the Magnetron.

\section{Magnetron Coupling}

In the proposed device a loop antenna inserted in a cavity resonator has been employed as power coupler, as shown in Fig. 3. Since the power extracted from the Magnetron is critically depended from the output circuit, the loop antenna must be designed in order to allow for the maxim power transfer. For the proposed device, the loop antenna terminates on a coaxial port with a characteristic impedance of $50 \Omega$. The port can be connected to an end-launcher for a waveguide which transforms such impedance of the TEM mode to the wave impedance of TE11 mode of a rectangular waveguide, if used for the power transfer.

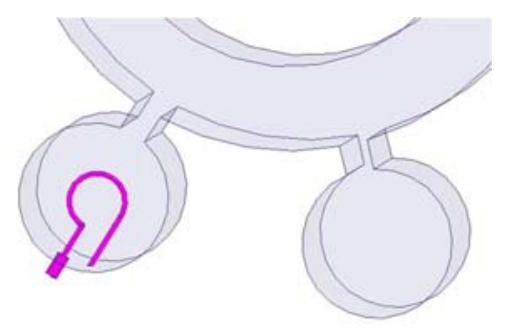

Fig.3. Magnetron loop power coupler inside a resonator

The efficiency and power output of a Magnetron depend on the resonant structure and the dc power supply.

The output cavity is a distributed resonant circuit characterized by an impedance $Z$ which can be schematized by a $R L C$ circuit. If the circuit receives energy at the NM resonant frequency, $L$ and $C$ resonates and the oscillation can be sustained only by compensating the loss on the cavity walls represented by $R$. In presence of such losses, the modes resonating not only at the eigenvalue frequency but in a frequency band, identifiable with the $3 \mathrm{~dB}$ bandwidth $\Delta f$. This band refers to the points where $\operatorname{Re}(\mathrm{Z})=$ $\operatorname{Im}(\mathrm{Z})$ which corresponds, on the Smith Chart, to two vectors with an argument of $45^{\circ}$ and an amplitude of $R / \sqrt{ } 2$. The unloaded quality factor $Q_{0}$ of the resonant circuit is defined as the ratio of the stored energy $W$ over the energy dissipated per cycle $P_{w}$ and can be calculated via the $3 \mathrm{~dB}$ bandwidth and the resonance frequency [23], as expressed by (5):

$$
Q_{0}=\frac{\omega W}{P_{w}}=\frac{f}{\Delta f}
$$




\section{ALBERTO LEGGIERI et al: MAGNETRON HIGH POWER SYSTEM DESIGN}

When the output coupler (the magnetic loop antenna) is connected to the cavity, it operates as a transformer, mutually coupling the resonator with an external load (or generator). A different impedance $Z$ ' is shown at the coupler port and the resonance changes. Such new resonance can be described by the loaded quality factor $Q_{L}$ which is the quality factor of the cavity when connected to a load (or a generator and measurement circuits). In this situation, if power $P_{\text {ext }}$ is extracted from the cavity (or supplied by a generator to the cavity), through the coupler, a portion of power $P_{w}$ is dissipated on cavity walls (i.e. on resistance R) and the same energy $W$ is stored in the cavity per cycle, the loaded quality factor is defined by (6):

$$
Q_{L}=\frac{\omega W}{P_{w}+P_{\text {ext }}}
$$

The quality factor that describes the degeneration of $Q_{0}$ due to the load (or to the generator and diagnostic impedances) connected to the coupler is the external quality factor, defined as $Q_{e x t}$ in (7).

$\frac{1}{Q_{L}}=\frac{1}{Q_{0}}+\frac{1}{Q_{\text {ext }}}$

The coupling coefficient $k$ is then defined as (8):

$k=\frac{Q_{0}}{Q_{\text {ext }}}=\frac{P_{\text {ext }}}{P_{w}}$

In the Smith chart, the impedance of a resonant circuit $Z(\mathrm{f})$, while varying $\mathrm{f}$, describes a circle. The radius $R_{c}$ of such resonance circle can describe the coupling strength. As mentioned above, when the system resonates, the sole power to sustain the loss on the walls is needed, a deficiency or an excess of power provided (or extracted) to (from) the cavity determines a mismatching between the cavity and the load (or generator).

If $0<k<1$, we have $R_{c}<0.25$ and the circle does not contain the center of the chart. This is the case of undercritical coupling. In this condition if a power $P$ is provided to the cavity, a portion of the incident wave is reflected while energy is storing inside the cavity, but the cavity is not being able to reach it maximum storable energy. If power is extracted from the cavity, it will be even less than the maximum obtainable power.

If $k=1$, we have $R_{c}<0.25$ and the circle is tangent to the center of the chart. This is the case of critical coupling, when maximum transfer of available power is ensured.

If $k>1$, we have $R_{c}>0.25$ the circle contains the center of the chart. This is the case of overcritical coupling. In this condition, if a power $P$ is provided to the cavity, a portion of the incident wave is reflected while energy is storing inside the cavity. Once the maximum energy is stored, the cavity even reflects power, due to the mismatch with the generator. Can be interesting to note that, if instantaneously the generator is switched off and the power supplying terminates, $P$ turns to zero and the cavity rejects the stored energy, providing towards the generator an instantaneous power which can be four times higher than $P$.

In order to compute graphically the quality factors and $k$, the detuned short position technique can be adopted: A transmission line is added between the output port of the coupler and the load (or generator). The circle is rotated around the origin due to the transmission line delay. From different frequency points in the chart $\Delta f, Q_{0}, Q_{L}$ and $Q_{e x t}$ can be determined as described in [22].

In the case of the resonant system of the Magnetron, the maximum circuit efficiency is obtained when the Magnetron is heavily loaded. Heavy loading, however, makes the tube quite sensitive to the load, which is undesirable in some cases. Therefore, the ratio of the unloaded quality factor and the external quality factor $Q_{L} / Q_{\text {ext }}$ is often chosen as a compromise between the conflicting requirements for high circuit efficiency and frequency stability [23].

However, for this design we have chosen to investigate on how to obtain the critical coupling between the output resonant cavity and the power coupler. In such case this values approaches to 0.5 and the coupling factor $k$ is unitary.

The dimension of the loop coupler can be estimated by observing the distribution of the magnetic field inside the resonator in the desired NM of operation. In order to extract the maximum power, the loop can be posed orthogonally to the microwave magnetic field lines and its area could be maximized without excessively perturb the resonator.

The in-frequency plot of $Z$ (f) can be computed through an electromagnetic simulator and the data can be analyzed in a mathematical simulator in order to find the coupling factor $k$ by employing an algorithm to calculate the detuned short position of $Z(\mathrm{f})$. For these analyses we have employed respectively HFSS and MATLAB.

\section{Dielectric Window Design}

\section{A. Dielectric Window Operating Principles}

Dielectric Windows are the devices which ensure atmosphere separation, while maintaining the microwave power transmission, between a power source and a load. These windows consist of a waveguide section with a dielectric disk inserted inside them.

Typically, energy transport system are based on rectangular waveguide $(\mathrm{RWg})$ and, due to technological reason, DWs are based on circular waveguide $(\mathrm{CWg})$ sections. For these reasons, the most common kind of window is the pill-box type. DW also enables the RWg to be filled with SF6 gas for high-power transmission $[8,19]$. 


\section{ALBERTO LEGGIERI et al: MAGNETRON HIGH POWER SYSTEM DESIGN}

The pill-box window consists of a CWg section of length $L$, at which centre a dielectric disk is inserted and at which ends $\mathrm{CWg}$ to $\mathrm{RWg}$ transitions are placed. One RWg face is connected to the high power microwave tube and the other is connected to the Load. The CWg subsection on the power tube side is in an UHV atmosphere and the subsection on the load side is filled by dry air or high dielectric strength gas, such as SF6.

The reason of the success of Pillbox windows resides in the circular profile, which is more suitable for fabrication of such devices: DW brazing process is carried inside a vacuum furnace with a typical maximum temperature of 780 ${ }^{\circ} \mathrm{C}$ in which the component materials are welded by a CUSIL alloy of $28 \% \mathrm{Cu}$ and $72 \% \mathrm{Ag}$. This temperature may deform the interface between the guide and the dielectric plate. Therefore, during this process, equally distributed forces have to be applied to the interface.

The described operation is easier to perform, by using circular symmetry, then using a rectangular one. From an analysis on commercial available dielectric materials, the alumina $\mathrm{Al}_{2} \mathrm{O}_{3} 97.6 \%$ has been chosen, which provides the high transparency to the microwave in order to obtain very low IL and great stress resistance. A Dielectric window for LINAC applications is shown in Fig. 4.

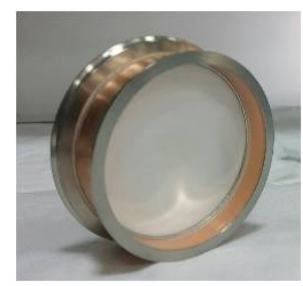

Fig.4. High transparent dielectric window for LINAC.

\section{B. Dielectric Window Design}

Due to the similarity of the dielectric constants of vacuum, air and SF6, could be possible to approximate the two $\mathrm{CWg}$ sectors as filled by the same dielectric. This simplification can be employed for a preliminary dimensioning of the device which must be optimized.

As shown in Fig. 5, since the CWg is partially filled with alumina ceramic gas and vacuum, this may be treated as equivalent to the $\mathrm{CWg}$ filled uniformly with a dielectric having effective relative permittivity $\varepsilon_{r}^{\prime}$.

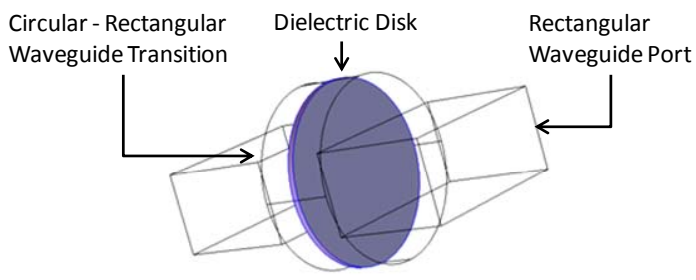

Fig.5. Pillbox Dielectric Window Layout.
Assuming the gas permittivity very similar to the vacuum, the effective permittivity of the whole $\mathrm{CWg}$ section may be evaluated as the average value with respect to the volumes occupied by the disk and the vacuum or gas, as expressed by the (9), where $L$ is the total length of the $\mathrm{CWg}$ section and $\varepsilon_{r}^{\text {disk }}$ is the relative permittivity of the dielectric medium of the disk.

$\varepsilon_{r}^{\prime}=1+\frac{t}{L}\left(\varepsilon_{r}^{\text {disk }}-1\right)$

For most of the high power pill-box windows, the value of $\varepsilon_{\mathrm{r}}^{\prime}$ will vary between 1.0 and 2.0. A value of 1.5 is reasonable for $\varepsilon_{r}{ }^{\prime}[11,19]$. Since the power transport system is based on $\mathrm{RWg}$, we can consider the wavelength of the fundamental mode in the rectangular waveguide as a known value $\lambda_{g R}$.

According with [19], the CWg radius which ensures the local transparency of the discontinuity can be found by the (10).

$$
r=\frac{\lambda_{0} p_{11}^{\prime}}{2 \pi \sqrt{1-\frac{\lambda_{0}^{2}}{\varepsilon_{r}{ }^{\prime} \lambda_{g R}^{2}}}}
$$

where $\lambda_{0}$ is the wavelength on free space, $r$ is the radius of the CWg and $p^{\prime}{ }_{11}$ is the first zero of the one order Bessel function derivative $\left(p_{11}^{\prime}=1.841\right)$. The wavelengths $\lambda_{0}$ and $\lambda_{g R}$ are calculated referring to the NM resonant frequency $f$ of the LINAC and, in particular, $\lambda_{g R}$ refers to the dielectric constant which fills the RWg.

Alumina disk should be designed with the minimum thickness $t$ which ensures the structural capacity to endure the direct stress $\sigma$ applied from the atmosphere normal force $F_{n}$, avoiding breakdowns or significant deformations. This direct stress, expressed in (11), corresponds to the pressure $\mathrm{P}$ which the dielectric cylindrical disk experiences on its area in contact to the UHV [19-20].

$\sigma=\frac{F_{n}}{\pi r^{2}}=P$

With a dielectric disk of $\mathrm{Al}_{2} \mathrm{O}_{3} 97.6 \%$ alumina with a plate radius of about 10-20 mm, a good thickness should be around $1 \mathrm{~mm}$. The total length of the $\mathrm{CWg}$ can be calculated in order to transfer maximum of microwave power at the junction by applying the $(12)[11,19]$.

$$
L=\lambda_{g R} \frac{\sqrt{\varepsilon_{r}{ }^{\prime}}}{2} \frac{b}{a}
$$


in which $a$ and $b$ are respectively the long and the short side of the RWg cross section.

The analytical design shown above is a simplification of the real dimensioning problem, in which can't be considered that some modes under cut off in the vacuum might be over cut off in the dielectric disk. For such reason, $L$ as well as $t$ must be optimized by an electromagnetic simulation.

\section{Dielectric Window Coupling}

The DW, when connected to the waveguide, needs to be matched to the wave impedance of the electromagnetic mode which propagates in the waveguide. Due to the future manufacturing production process, global characteristics of the structure composed by the LINAC and its own dielectric window will be degraded by the welding processes of the DW. For a standing wave LINAC, a tuning strategy is possible, since it resonates at a specific NM frequency, which is to be matched with the NM frequency of the Magnetron.

After fabricating and welding the pill-box window section to the LINAC Power Coupler (LPC), reflection coefficient $\rho_{\text {in }}$ ' can be measured and the opportune values of the matching elements can be obtained. For this reason, the IMN has been made by an iris loaded waveguide, which is easily realizable, perfectly according to the exact NM working frequency of the brazed LINAC, on which the DW will be mounted $[19,26]$.

At the NM frequency of resonance of the LINAC, without the window, the LPC shows the External Reflection Coefficient $\rho_{\text {Ext }}$ to the outside; $\rho_{\text {Ext }}$ consists of the LINAC reflections coefficient $\rho$, transformed by the LPC. A pill-box DW is connected to the LPC and $\rho_{\text {Ext }}$ is transformed to $\rho_{\text {in }}$, by the window. This connection is performed by a brazing process. After brazing, $\rho_{\text {in }}$ ' can be measured and an opportune Input Matching Network (IMN) can be designed. The IMN transforms $\rho_{\text {in }}$ ' in $\rho_{\text {in }}$ by placing it to the centre of the Smith chart (Fig. 6), providing the right matching condition between the LPC and the HPMW source and ensuring the optimum reflection coefficient $\rho_{\text {Opt }}$ shown by the LPC to the LINAC (Fig. 7). $\rho_{\text {Opt }}$ is the reflection coefficient that makes unitary the coupling factor $\beta$ of the LPC, which produce the LINAC critical coupling with its input port $[19,26]$.

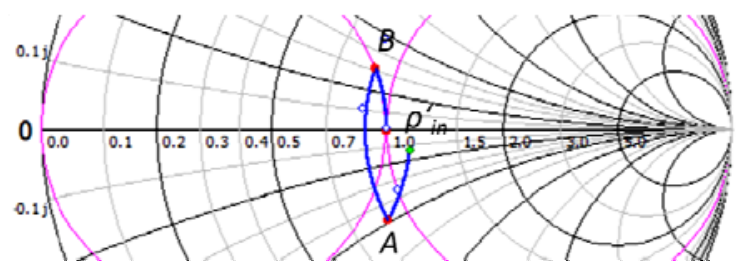

Fig. 6. Single frequency lumped IMN example: From the initial position (green point), $\rho_{\text {in }}$ ' is moved by a shunt capacitor to the red point $A$ from which is moved to the point $B$ by a series inductor. Another shunt capacitor moves the reflection coefficient from B to the center of the Smith Chart.

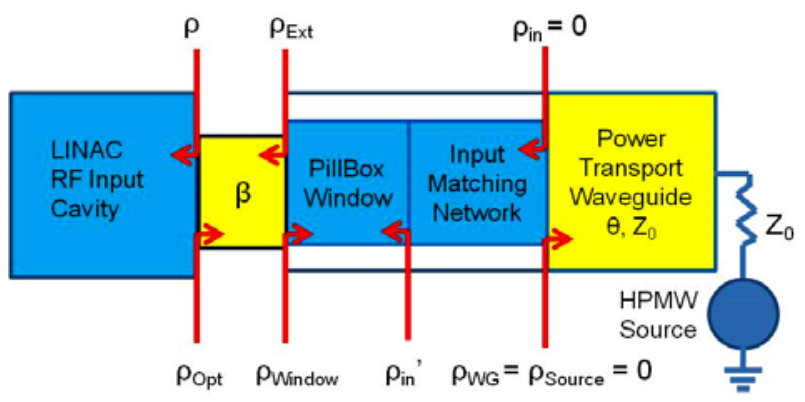

Fig. 7. Devices disposition and reflections coefficients at the interfaces.

In order to physically realize the $\mathrm{DW}$, the $\mathrm{CWg}$ sections are welded at both the two interfaces: one (at the UHV side) welded to the LINAC and the other (at the normal atmosphere side) to an RWg flange. At this flange the transport $\mathrm{RWg}$ will be connected by screws and the reflection coefficient $\rho_{\text {in }}$ ' can be measured at the $\mathrm{NM}$ resonant frequency of the LINAC.

Let us consider, for example, the case in which the cascade of the LINAC and DW shows an input reflection coefficient $\rho_{\text {in }}$ ' on the fourth quarter of the Smith Chart (the green point in fig. 6). In order to perform a correct matching $\rho_{\text {in }}$ ' is to be moved to the center. For this aim, we propose a matching technique, according to theory described in [24].

Due to the narrow band character of standing wave LINAC, the system can be matched at the LINAC NM frequency of resonance, by introducing the lumped IMN shown in Fig.8. It consists of $\pi$-network with a first shunt capacitor followed by a series inductor and by another shunt capacitor.

The Matching path performed by this network is the same shown in fig. 6: the first shunt capacitor moves $\rho_{\text {in }}$ ' from its initial position to the point $A$, near the real axis, and the series inductor moves $A$ to the circle at unitary conductance in the point $B$. The second shunt capacitor moves $\mathrm{B}$ to the center of the Smith Chart which is the desired point $\rho_{\text {in }}$ '. This operation can be performed manually or by using a circuital simulator. For such kind of operation, we have employed PASAN (see Fig.6) and AWR Microwave Office (MWO) (see fig.8), equivalent for this analysis.

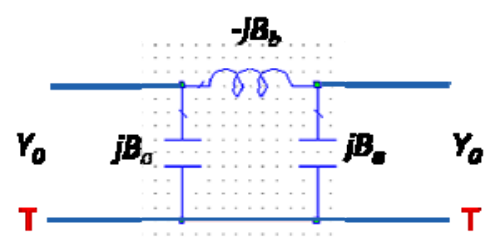

Fig. 8. Lumped IMN Example: A capacitive iris equivalent circuit.

In order to realize such network with a waveguide discontinuity is to be considered the structure of a capacitive iris, which can be dimensioned as widely described in [25]. Capacitive iris structure is represented in Fig. 9 and its 


\section{ALBERTO LEGGIERI et al: MAGNETRON HIGH POWER SYSTEM DESIGN}

sections are described in Fig.10, in which the obstacle is inserted at the reference planes $T$. In the wavelength range expressed in (13):

$$
\frac{b}{\lambda_{g}}<1
$$

the capacitive iris has an Equivalent circuit, at the planes T, which is the same shown in Fig. 8. An application of such matching principle has been shown also in [26]. In order to simplify the factory process, the thickness can be set to a commercial RWg gasket in which the iris can be physically realized.

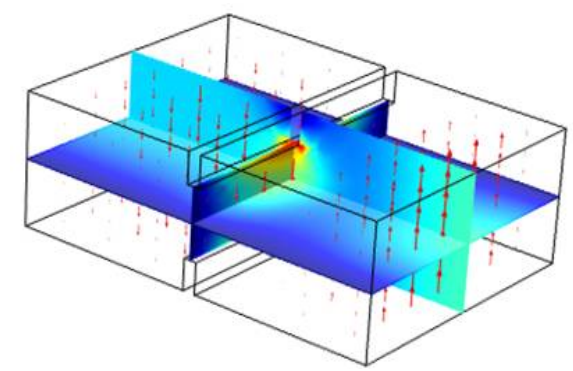

Fig. 9. Capacitive iris structure on a RWg sector and fundamental mode $\left(\mathrm{TE}_{10}\right)$ electric field distribution on bisecting planes.

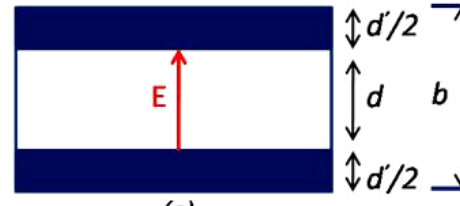

(a)

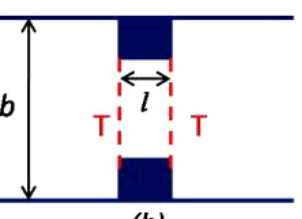

(b)
Fig. 10. Capacitive iris: longitudinal section (a), and front (b) views.

Only shunt elements can be usefully employed in order to match the accelerating structure by using waveguide based devices. The design procedure of this IMN refers to a LINAC with an external reflection coefficient shown by the LPC $\rho_{\text {Ext }}$ located on the third quadrant of the Smith Chart.

The capacitive iris is well suitable for this condition and it can be employed for several configuration, however if some difficulties are encountered, other strategies can be adopted as for example inductive iris.

As described in [25], waveguide inductive iris has a shunt inductance behavior. Furthermore an hybrid matching can be adopted as described below: For example, if $\rho_{\text {in }}$ ' is located in the first or in the fourth quarter of the Smith Chart, we can move $\rho_{\text {in }}$ ' to an opportune $\rho_{c}$ located in the third quarter of the Smith Chart by adding a transmission line. Since the reflection coefficient applied to the line is given by (14):

$\rho_{i n}^{\prime}=\left|\rho_{i n}^{\prime}\right| e^{\operatorname{Arg}\left(\rho_{\text {in }}^{\prime}\right)}$ the line has to introduce a phase shift given by (15) [12]:

$$
\vartheta=\operatorname{Arg}\left(\rho_{\text {in }}^{\prime}\right)-\operatorname{Arg}\left(\rho_{c}\right)
$$

The line can be realized with a standard RWg section operating in the fundamental mode, with a $\mathrm{TE}_{11}$ mode wave impedance $Z_{0}$. The $\mathrm{RWg}$ length can be found from the relation of phase shift (16) [24]:

$$
\vartheta=\frac{2 \pi}{\lambda_{g R}} l_{R}
$$

After this phase shift, $\rho_{\mathrm{c}}$ can be moved to the centre of the Smith Chart by a capacitive or an inductive iris.

\section{Dielectric Window Multiphysics Aspects}

The power dissipation due to the Joule effects related to the high power in transit inside the DW produces a considerable temperature increase.

Such temperature receives an additional contribution by the heating of the microwave device at which is connected, principally by thermal conduction through the connection faces.

The temperature increase induces a thermal expansion of the whole structure, which may results in high surface losses and displacements which may alter the desired EM behavior of the device, decreasing performance.

We show how is possible to mitigate the degradation of the performances induced by the thermal losses by choosing a device shape and material which, when deformed by its temperature, modify constructively the EM fields reincreasing performances.

For this aim, CWg sector is made by copper and brazed on a $1 \mathrm{~mm}$ thickness ring of steel which is welded to a $\mathrm{RWg}$ flange of opportune dimensions.

\section{MAgnetron COMPUTATIONAL Model}

Magnetron Simulation has been performed through the Finite Element Method (FEM). General purpose software COMSOL Multiphysics have been employed to couple TS, EM, ES and CPT analysis by Moving Mesh (MM) dedicated interface and storing temperature information. MM computation moved the mesh in function of the displacement computed by the TS analysis.

Subsequently, EM, ES and CPT computations have been executed on the new meshes and by receiving the temperatures evaluated by the TS study [17-18].

In order to simplify the model, cathode surface is modeled as a copper layer. High vacuum medium between anode and cathode is air at pressure of $P=10^{-10}$ bar.

An FEM Electromagnetic simulation of the proposed Magnetron with the magnetic loop power coupler has been performed by using HFSS of ANSYS-ANSOFT. 


\section{ALBERTO LEGGIERI et al: MAGNETRON HIGH POWER SYSTEM DESIGN}

In such ambient, a Driven Modal (DV) electromagnetic simulation has been performed to define the optimal geometry of the power coupler.

\section{A. Thermo-structural Analysis}

TS analysis is employed to solve the system of steady state stress and heat equations (17) [17-18] on COMSOL:

$$
\begin{aligned}
& -\nabla \cdot \sigma_{S}=\bar{F}_{V} \\
& \rho C_{p} \bar{u} \cdot \nabla T=\nabla \cdot(k \nabla T)+Q
\end{aligned}
$$

where $\sigma_{\mathrm{S}}$ is the stress and $\bar{F}_{V}$ is the force per unit volume; $\rho$ is the density, $C p$ the heat capacity and $k$ the thermal conductivity of the material. $Q$ is the heat source, $\bar{u}$ is the velocity vector.

Since temperatures are dependent to the displacements, these two equations are solved simultaneously. The cathode represents a constant volume heat density source made by tungsten of $Q=0.405 \mathrm{GW} / \mathrm{m}^{3}$. The lateral external metallic surfaces of anode and the bases of the cathode cylinder are connected to rigid supports, so that represent fixed surface constraints. These constraints allow the compressive forces induced by the thermal expansion. The external lateral surfaces are typically cooled by fluid held at $35^{\circ} \mathrm{C}$.

\section{B. Electromagnetic Waves}

The EMW computation, which is applied only to the interaction region, solved the wave equation in the frequency domain (18) [17-18] on COMSOL.

$$
\nabla \times \mu_{r}^{-1}(\nabla \times \bar{E})-k_{0}^{2}\left(\varepsilon_{r}-\frac{j \sigma}{\omega \varepsilon_{0}}\right) \bar{E}=0
$$

where $\mu_{r}$ is the magnetic permeability, $\varepsilon_{r}$ the electrical permittivity and $\sigma$ the electrical conductivity of the material; $\varepsilon_{0}$ is the electrical permittivity of the vacuum, $k_{0}$ the wave number in free space, $\omega$ the wave angular frequency and $\bar{E}$ the electric field.

The surfaces are modeled considering the material losses. Surface bases of the interaction region are modeled to be transparent for scattered wave; hence potential resonance errors are avoided.

\section{Electrostatics}

ES analysis is employed to solve the system of electrostatics equations (19) [17-18] on COMSOL.

$$
\begin{aligned}
& \nabla \cdot \bar{D}=\rho_{V} \\
& \bar{E}=-\nabla V
\end{aligned}
$$

where $\rho_{V}$ is the volumetric charge density, $\bar{D}$ the dielectric displacement vector and $\mathrm{V}$ the voltage. The anode electric potential is set to zero and the cathode electric potential to $V_{k}=-60 \mathrm{MV}$.

\section{Charged Particle Tracing}

CPT analysis is employed to calculate the particle trajectories considering the ES computation, by solving the (20) [17-18] on COMSOL.

$\frac{\partial\left(m_{p} \bar{v}\right)}{\partial t}=\bar{F}_{t}$

where $m_{p}$ and $\bar{v}$ are respectively the particle mass and velocity, $\bar{F}_{t}$ the total force experienced by the particle.

Since the cathode current density is constant in time and on the cathode surface, the charge release is represented by a short pulse sequence with initial null value, so that the number of particle per release is given by (21):

$$
N=\frac{I \Delta t}{e}
$$

where $I$ is the design cathode current, $e$ the elementary charge of the electron and $\Delta t$ is the time interval between two consecutive charge releases of $N$ particles.

In order to decrease computational costs, this number has been set to $N^{1}=123$ and a charge multiplier factor $n=10^{8}$ has been added in order to respect the space charge effect. The charge multiplication factor can be calculated as $n$ $=N / N^{l}$. The electric part of the Lorentz force $F=e(-D V)$ includes the voltage calculated iteratively by the ES analysis. Magnetic part of the Lorentz force $F=e v \wedge B$ includes the desired magnetic induction $B$ [17-18]. Anode walls are represented to absorb negative charges.

\section{E. Power Coupler Optimization}

The DV electromagnetic computation has been applied only to the interaction region and performed in the frequency domain on HFSS.

The aim of this analysis is the optimization of the system composed by the Magnetron and the power coupler. The simulation is based on the resolution of the equation (18) [17-18] in the stationary regime in a small band near the $\pi$ mode frequency of resonance $f$. The optimization has been performed by sweeping the value of the coupler penetration $d$ inside the output resonant cavity while calculating the scattering parameters at the coaxial port.

For the kind of calculator we have employed, for the EM analysis of the system which includes the power coupler, the computation through HFSS could be faster. This is due principally to the $\mathrm{C}++$ core of HFSS which needs fewer resources in our calculator than COMSOL, which operates 


\section{ALBERTO LEGGIERI et al: MAGNETRON HIGH POWER SYSTEM DESIGN}

in the JAVA virtual machine. We specify that these software applications are equivalent and deserve of the same consideration in term of quality and reliability.

\section{DiELECTRIC Window COMPUTATIONAL MODEL}

For a complete Multiphysics modeling of the DW, thermo mechanical computation, including the joule effect, and EM analysis have been independently performed in the effective spaces in which respectively structural stresses and EM waves must be computed. Then, by considering the thermo structural alteration, a mesh modification has been applied to the whole space, including the region of the EM computation, in order to evaluate the EM behavior in the deformed geometry with the altered temperature.

The solver is organized in performing the following steps: First, a FD EMW analysis has calculated the EM fields in the DW by receiving the Magnetron output power at the input port. This study is applied to the dielectrics.

Subsequently, a stationary analysis to compute the joule effects by receiving the surface currents related to the previous electric field previously computed.

Joule effects have been evaluated only on the DW carrier walls and alumina excluding air and vacuum, since the currents are on the surfaces shared between carrier walls and dielectrics.

Together with the currents, this analysis take in input the temperature of the surface connected to the Magnetron or LINAC.

This temperature has been set to $35^{\circ} \mathrm{C}$, since many vacuum tubes such as Magnetrons or Klystrons and also LINAC's are thermostated at this value. Moreover, in LINAC applications, source and load share the same cooling system.

Displacements are received in input by the MM calculation in order to move the meshes of the whole system, including the vacuum volume, in which the thermo mechanical analysis has not been performed.

Afterwards, by a stationary analysis, the electric fields and the scattering parameters are calculated. The ultimate EM analysis has been performed on these moved meshes, considering the temperatures computed by JHTE study. Such analysis is applied only to the dielectrics and DW carrier walls are excluded.

The wave equation in the frequency domain (18) has been solved again in this step. Such analysis is obliviously applied only to the dielectrics; copper and steel volumes are excluded.

Due to the simplicity of the geometry, default settings have been adopted, by choosing Physic controlled mesh with finer element size. The complete mesh consists of 28806 elements and the minimum element size is $0.65 \mathrm{~mm}$, which corresponds to $0.02 \lambda_{0}$.

\section{A. Electromagnetic Waves}

Due to the Narrow Band character of the Magnetron, operating at $9 \mathrm{GHz}$, the EM FD analysis of the DW has been performed between 8.9 and $9.1 \mathrm{GHz}$.

The wave equation in the frequency domain (18) has been computed.

\section{B. Dielectric Window Numerical Optimization}

Even if the DW is designed for coupling a LINAC and Magnetron with very narrow bandwidth, is opportune to simulate and optimize in a bit larger band.

The solution is computed by a Stationary solver on the equation (7) at the operative frequency $f$, by sweeping the length $L$ and the thickness $t$ and calculating the scattering parameters at two ports.

The simulation has been set with the DW values analytically calculated above, $L=13 \mathrm{~mm}$ and $r=12 \mathrm{~mm}$, and an $\mathrm{Al}_{2} \mathrm{O}_{3} 97.6 \%$ plate of thickness $t=1 \mathrm{~mm}$. This alumina presents a dielectric constant of $\varepsilon_{\mathrm{r}}=9.0$ in X band, which in the simplified analytical dimensioning has not been considered properly.

For the proposed X-Band Magnetron, operating at $f=9$ $\mathrm{GHz}$ and driven through a RWg WR90, (which have $a=$ $22.86 \mathrm{~mm}$ and $b=10.16 \mathrm{~mm}$ ) with $\varepsilon_{r}=1$ and considering that at $9 \mathrm{GHz}$ we have $\lambda_{g R}=49 \mathrm{~mm}$ and $\lambda_{0}=33 \mathrm{~mm}$, the DW can be dimensioned as follows: By applying the (10) with $\varepsilon_{r}{ }^{\prime}=1.5$ we obtain a radius of $r=12 \mathrm{~mm}$. From the (12) results that the total length of the CWg is $L=13 \mathrm{~mm}$.

By introducing this value in a numeric computation, we obtained an accurate dimensioning of the structure. Optimization has shown an optimum length of the circular sections $L=14 \mathrm{~mm}$ and optimal dielectric plate thickness $t$ $=0.7 \mathrm{~mm}$, as reported in Fig. 11 .

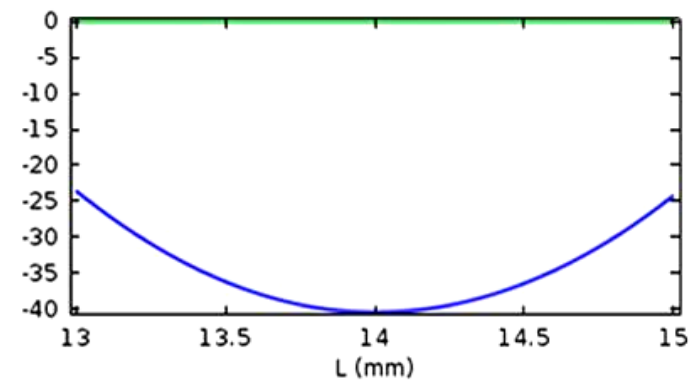

Fig. 11. Simulated $S_{11}$ (blue trace) and $S_{21}$ (green trace) in $\mathrm{dB} @ 9 \mathrm{GHz}$ versus the CWg length $L(\mathrm{~mm}), \mathrm{S}_{11}=-40 \mathrm{~dB}$ for $L=14 \mathrm{~mm}$ and $t=0.7 \mathrm{~mm}$.

\section{Joule Heating and Thermal Expansion}

The JHTE analysis is employed to solve the system of steady state equations (17) and (21) [18]:

$$
\begin{aligned}
& \nabla \cdot \bar{J}=\bar{Q}_{J} \\
& \bar{J}=\sigma \bar{E}+\bar{J}_{E}
\end{aligned}
$$




\section{ALBERTO LEGGIERI et al: MAGNETRON HIGH POWER SYSTEM DESIGN}

where; $J$ is the current density, $\bar{Q}_{J}$ the current source, $\bar{J}_{E}$ the external current density. The non ideal vacuum and the air atmospheres inside the DW volume are modeled only to describe the heat transfer and excluded from moment computations. Since this DW can be connected between the Magnetron and the WG or between the LINAC and the WG, the external metallic surface of DW input port is locked to the rigid structure of one of these devices and the other to a WG [8-11]. Thus it represents a fixed constraint.

Magnetrons and LINAC's, are cooled by fluid held at $35^{\circ} \mathrm{C}$, consistently with many typical Magnetrons and LINAC's cooling systems.

\section{Magnetron Simulation Results}

\section{A. Thermo-Mechanical Features}

By imposing the designed heat density, the TS stationary analysis has shown a maximum temperature of $1051^{\circ} \mathrm{C}$ on the cathode, according with the thermo-electrical threshold for cathode electron release. This result is shown in the left image of Fig. 12.

Maximum stress is about $6.0 \mathrm{GN} / \mathrm{m}^{2}$. Maximum total displacement is located on lateral cathode surface, and is about $0.13 \mathrm{~mm}$ as shown in right image of Fig. 12 .
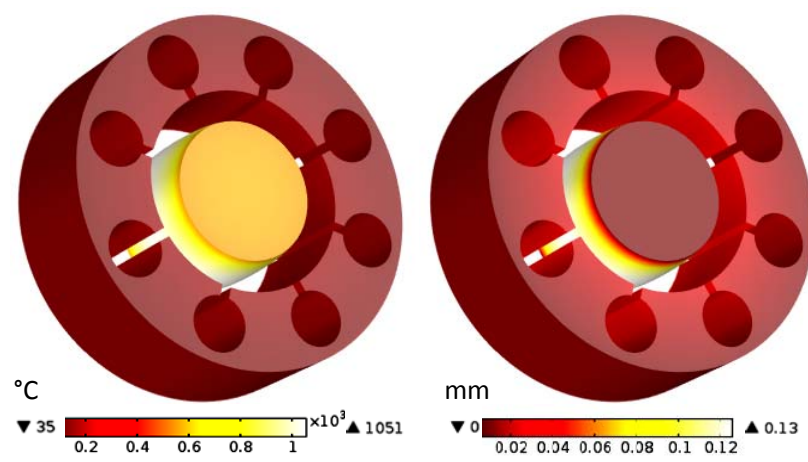

Fig.12. Temperature, ${ }^{\circ} \mathrm{C}$ (left) and Displacement, mm (right).

\section{B. Resonances}

The simulation output shows the field power density distribution of the resonant modes in the transversal cross section of the Magnetron.

The search of normal modes has been supported by superposing, on the same streamline graphic, a set of vectors composed by the $x$ and $y$ components of the electric field at the center of the cavities. Resonant fields in working condition are plotted in Fig. 13. Arrows represent the electric field direction inside cavities.

The resulting Magnetron EF's and Quality factors in cold conditions are: $f_{2 \pi}=3.673 \mathrm{GHz}, f_{\pi}=9.061 \mathrm{GHz}$ and $Q_{2 \pi}=$ $1000, Q_{\pi}=8300$.
In thermo mechanical operative conditions, the displacement and the electrical properties alteration cause a variation of the Eigen-frequencies to: $f_{2 \pi}=3.663 \mathrm{GHz}, f_{\pi}=$ $9.042 \mathrm{GHz}$ and a variation of the quality factors to: $Q_{2 \pi}=$ $950, Q_{\pi}=8250$.
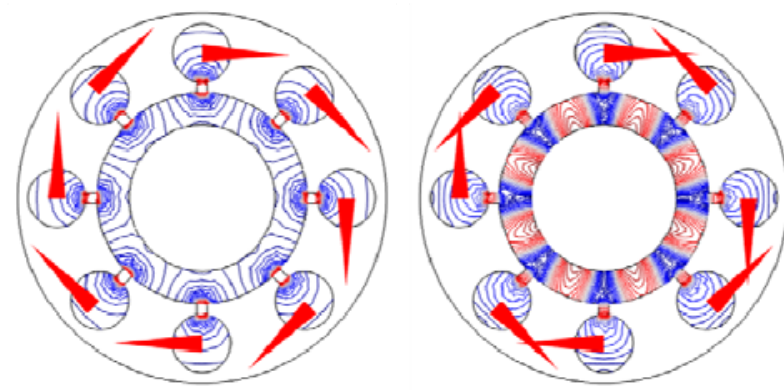

Fig.13. E field in working conditions: $2 \pi$ mode (left) and $\pi$ mode (right).

\section{Electrostatic Fields and Particle Tracing}

The resulting maximum electrostatic fields are: In cold conditions: $E_{\operatorname{Max}}=7.94 \mathrm{MV} / \mathrm{m}$, and in thermo mechanical operative conditions: $E_{\operatorname{Max}}=8.03 \mathrm{MV} / \mathrm{m}$.

The thermal excitation contributes to increase the maximum particle velocity magnitude from $v_{\text {cold }}=1.196 \cdot 10^{8}$ $\mathrm{m} / \mathrm{s}$ in cold conditions, to $v_{\text {hot }}=1.227 \cdot 10^{8} \mathrm{~m} / \mathrm{s}$ in thermo mechanical operative conditions.

Particle trajectories, at $t=2.16 \mathrm{~ns}$ after cathode release, in working conditions are depicted in the left image of Fig. 14.
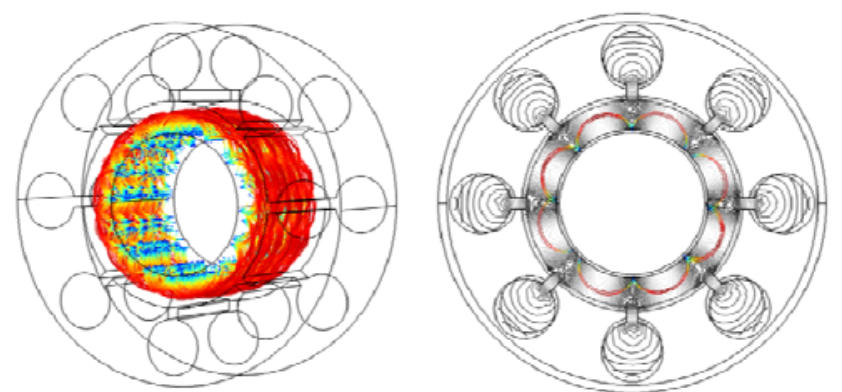

Fig.14. 3D plot of the particle trajectories (Left) and cross sectional view for working point identification (Right).

\section{Working Points Computation}

Working points are the couples of $V$ and $B$ which ensures the correct superposition $[13,17]$ of particle trajectories with the desired resonant electric field. These ones have been plotted on a cross plane and, by observing their superposition, the correct operative values of $V$ and $B$ have been evaluated. In order to reduce computational complexity, a PT simulation has been performed with null initial velocity. Electrons traveling in crossed electric and magnetic fields follow cycloidal paths which have cusps if their initial velocity is null [21]. 
By setting the static conditions of $\pi$ mode operation and null initial velocity, particle trajectories have been plotted. A superposition of these trajectories with $\pi$ mode oscillating field shows a correct synchronism condition, reported in the right image of Fig. 14. In the graphic of Fig. 15, theoretical working points are plotted: cathode voltage on the horizontal axis and axial magnetic induction on the vertical axis. However some points are not useful because of their excessive voltages.

$B(T)$

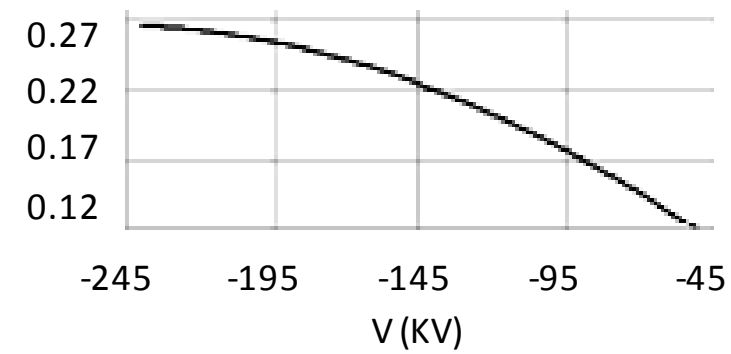

Fig.15. Magnetron $\pi$ mode theoretical working points

\section{E. Power Coupler Optimization}

The analysis of the Magnetron power coupling has been performed on ANSYS-ANSOFT HFSS. The presence of the power coupler produces a frequency shift due to the introduction of material inside the cavity. For different values of the coupler penetration $d$ inside the cavity, the frequency of resonance moves of some $\mathrm{MHz}$. For the optimum value of $d$, the system shows a return loss $\mathrm{RL}=30$ $\mathrm{dB}$ and the radius of the plot in resonance tends to the unity, approaching the critical coupling as shown respectively in Fig. 16 and 17.

The values of the reflection parameters in the frequency bandwidth have been exported from ANSYS-ANSOFT HFSS and elaborated through a custom MATLAB code, computing the quality factors by the detuned short position technique described in [22]. The output is shown in figure 18 in which, for the optimum value of $d$, the value of $k$ is 1.054 .

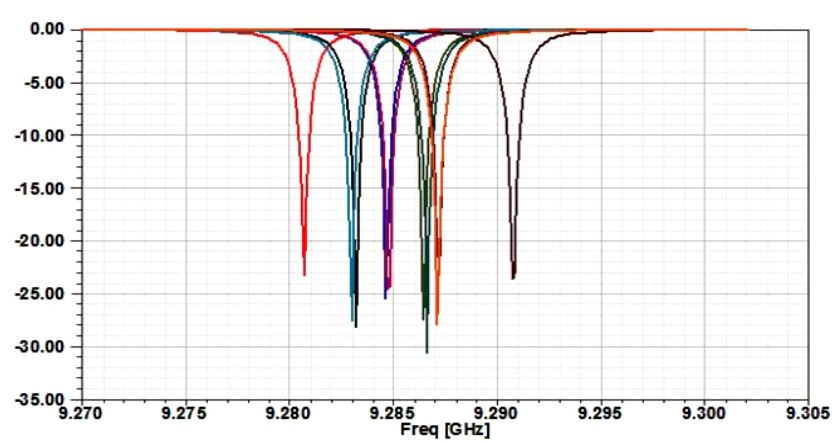

Fig.16. dB magnitude of the Scattering reflection parameter of the system for several values of $d$.

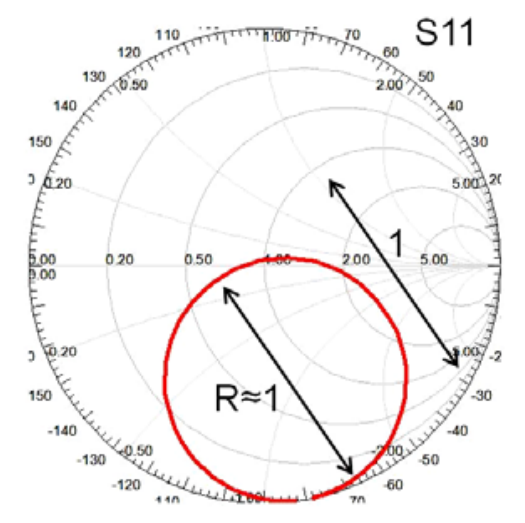

Fig.17. Scattering reflection parameter of the system on the Smith Chart. For optimum value of $d$ the Magnetron is critically coupled.

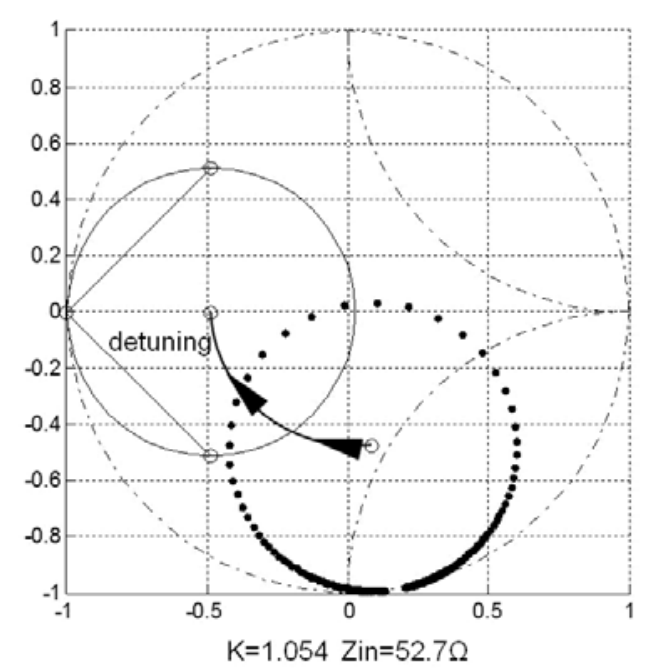

Fig.18. MATLAB output of the scattering reflection parameter on the Smith Chart in detuned short position.

\section{Dielectric Window Simulation RESUlts}

The proposed DW has been designed to operate by receiving the Magnetron power output, which has been rounded up to a maximum power of $P_{\text {peak }}=3 \mathrm{MW}$ pulsed with a duty Cycle of $\delta=0.004 \%$. By imposing a Power input of average power $P_{\text {ave }}=\delta \cdot P_{\text {peak }}=120 \mathrm{~W}$.

\section{A. Surface Currents and Thermo-Mechanical effects}

The EM FD stationary analysis has shown a maximum surface current density of $I_{s}=58.3$ A, reported in Fig.19. The temperature distribution is shown in the upper image of Fig.20. In order to underline the deformation, stress and displacement are been plotted with a magnified scale. In the following figures, black outlines represent the original conformation, and the stained volume represents the deformed structure. The maximum stress is located at the junctions between the RWg and the flanges which connect the circular section of the DW and is about $0.14 \mathrm{GNm}^{-2}$. 


\section{ALBERTO LEGGIERI et al: MAGNETRON HIGH POWER SYSTEM DESIGN}

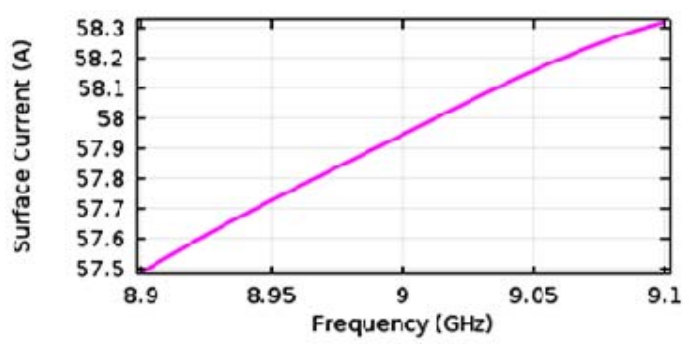

Fig. 19. Surface Current Amplitude versus Frequency.

The maximum total displacement is located on the flange surface connected to the vacuum tube interface; this result is due to the further heating of the power tube. Such maximum displacement is about $10 \mu \mathrm{m}$ and is shown in the lower image of Fig.20.
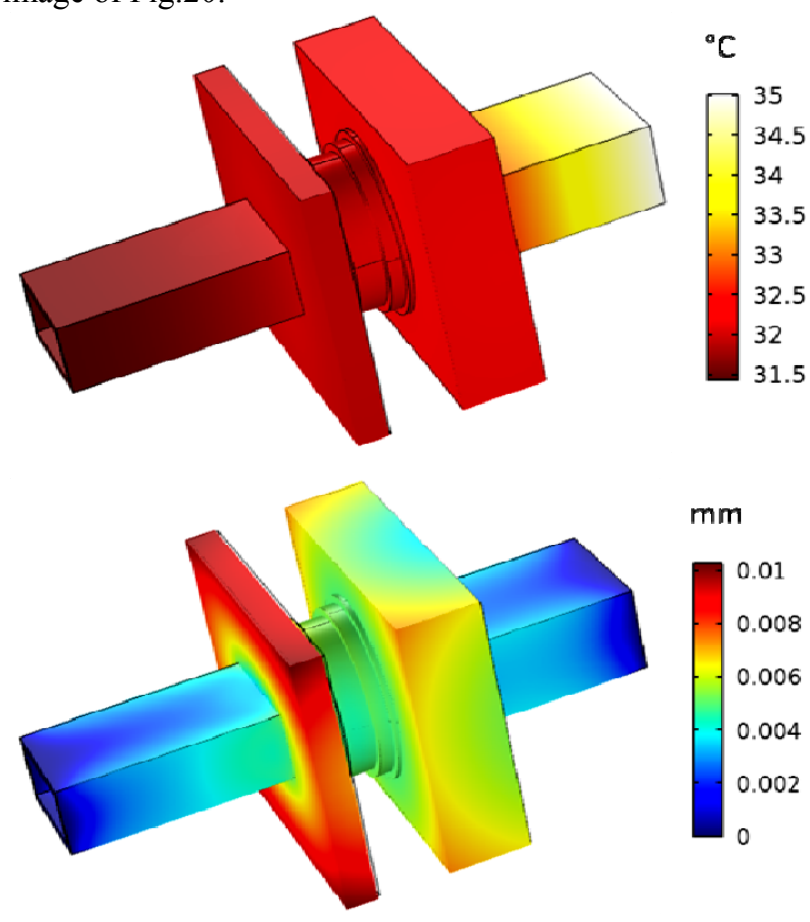

Fig. 20. Temperature (Upper) and Displacement (Lower).

\section{B. Electric fields and Scattering Parameters}

The simulation output shows the electric fields in operating stage, reported in Fig. 21.

Reflections and Transmission Scattering parameters at the DW ports have been computed in different thermomechanical conditions and represented in Fig.22 and 23. In these figures the parameters related to the cold conditions are represented by blue curves labeled "cold"; the parameters related to the exclusive thermal effect are represented by the pink curves labeled "Only Thermal"; the parameters related to the whole thermo-mechanical operation are represented by green curves labeled "Thermal Deformed".
In the proposed device, the displacements exert a constructive effect. Note that, by adopting the multiphysics modeling based design of the DW, we have ensured the mitigation of the power losses due to the surface currents. This result is evident by observing the improvement of the scattering parameters when the deformation is considered hence the EMW analysis is performed on the deformed meshes.

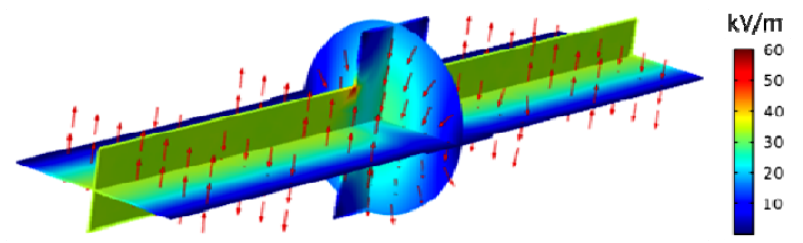

Fig. 21. Electric field cross sectional view in working conditions.

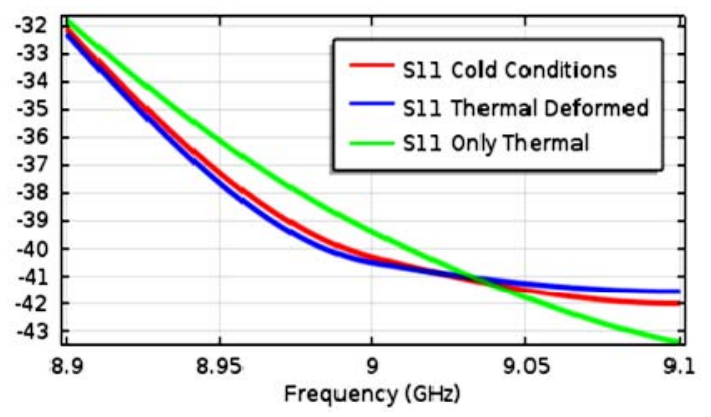

Fig. 22. Scattering Reflection Parameter, dB Magnitude.

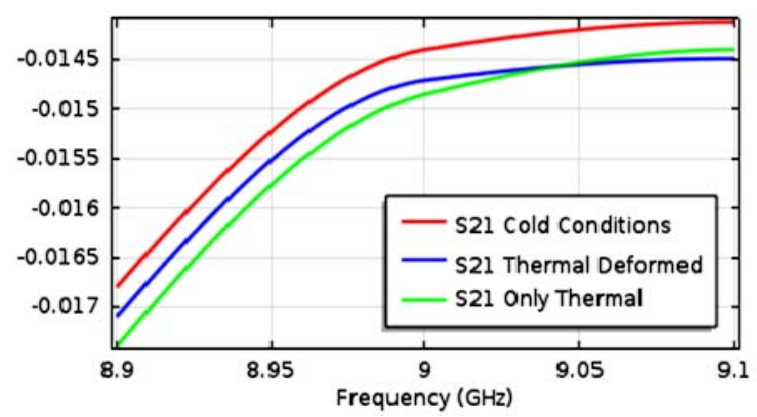

Fig. 23. Scattering Transmission Parameter, dB Magnitude.

\section{CONCLUSIONS}

In this study a particular design technique of an X-Band Magnetron to LINAC High Power Transfer System based on an 8 slots resonant cavities Magnetron and a dedicated Vacuum Dielectric Window has been proposed. A main analytical design of devices is shown with compact formulas and is followed by numerical optimization. Electromagnetic and Thermo-mechanical coupling between these devices is described, particular attention has been given to the Magnetron power coupling and DW matching.

A Multiphysics simulation has been performed in order to consider thermal-structural effects due to the cathode 


\section{ALBERTO LEGGIERI et al: MAGNETRON HIGH POWER SYSTEM DESIGN}

heating for the Magnetron and the Joule effect for the DW. The thermo-mechanical contact and thermostatation between these devices and LINAC has been considered. Magnetron resonance and particle tracing have been studied, and many aspects have been investigated through a Multiphysics modeling.

By considering the thermo-mechanical effects due to the cathode heating, Magnetron particle motion and $\pi$ mode working points have been computed.

For the coupled structure, the Magnetron shows a return loss $R L=30 \mathrm{~dB}$ and a coupling factor $k=1.054$, ensuring the maximum power extraction from the resonant structure. By applying the design condition of $V=60 \mathrm{KV}$ and $B=1330$ $\mathrm{G}$, in order to have $I=110 \mathrm{~A}$; this Magnetron, with a typical efficiency of $40 \%$, can produce a pulsed microwave peak power of $2.64 \mathrm{MW}$.

DW scattering parameters and electric fields have been documented, by considering the electromagnetic heating due to power dissipation through the DW. The proposed DW can ensure a minimum return loss of $R L=32 \mathrm{~dB}$ with a maximum Insertion Loss $I L=0.017 \mathrm{~dB}$ when it is carrying a pulsed power of $P_{\text {peak }}=3 \mathrm{MW}$ with a Duty Cycle of $\delta=$ $0.004 \%$ provided by the X-Band Magnetron. It operates at the center frequency $f=9 \mathrm{GHz}$ with $200 \mathrm{MHz}$ of Bandwidth.

In this study several modeling strategies have been shown in order to save computational resources. We have shown how to mitigate the degradation of the performances, induced by the thermal losses, by choosing a device shape which, when deformed by its temperature, modify constructively the EM fields to re-increase performances. Different software applications have been employed: COMSOL, HFSS, AWR MWO, PASAN, and MATLAB.

According to this study, the appropriate materials have been chosen in order to ensure the correct operation of the device in thermal stress critically affected working conditions.

\section{REFERENCES}

[1] B. Levush, D.K. Abe, J.P. Calame, B.G. Danly, K.T. Nguyen, E.J. Dutkowski, R.H. Abrams, R.K. Parker: "Vacuum Electronics: Status and Trends", 2007 IEEE Radar Conference, p:971-976.

[2] C.M. Armstrong: "The vitality of vacuum electronics", IEEE 14th International Vacuum Electronics Conference (IVEC), 2013, p:1-3.

[3] J.H.Booske, R.J.Dobbs, C.D.Joye, C.L.Kory, G.R.Neil, P.Gun-Sik, P.Jaehun, R.J.Temkin: "Vacuum Electronic High Power Terahertz Sources", IEEE Transactions on Terahertz Science and Technology, Vol:1, Issue: 1, 2011, p:54-75.

[4] J.X.Qiu, B.Levush, J.Pasour, A. Katz, C.M. Armstrong, D.R. Whaley, J. Tucek, K. Kreischer, D. Gallagher: "Vacuum tube amplifiers", IEEE Microwave Magazine, Vol:10, Issue:7, 2009, p:3851.

[5] V.F. Vikulov et Al., "A Compact Standing-Wave Electron Linac with RF Drive System Using 3 dB Hybrid Junction," IEEE Transactions on Nuclear Science, Volume 26,Issue 3, 1979, pp. 4292 - 4293.
[6] A. Saverskiy, H. Deruyter, M. Hernandez, A. Mishin and D. Skowbo, "Portable X-Band Linear Electron Accelerators for Radiographic Applications," Proc. Particle Accelerator Conference, 2005, pp. 1985 $-1987$.

[7] S. M. Hanna, "Role of Microwave Accelerators in Cancer Treatment," Proc. Microwave Symposium, 2007. IEEE/MTT-S International, 2007, pp. 1337 - 1340.

[8] H. Arai, N. Goto, Y. Ikeda and T. Imai, "An Analysis of a Vacuum Window for Lower Hybrid Heating," IEEE Transactions on Plasma Science, vol. PS-14, No. 6, 1986, pp. 947-954.

[9] K. Hirano, Y. L. Wang, T. Emoto, A. Enomoto and I. Sato "Development of a High Power 1.2MW CW L-Band Klystron," Proc. Particle Accelerator Conference, Dallas, TX, 1995, pp. 1539 - 1541.

[10] Y. Otake, S. Tokumoto and H. Mizuno, "Design And High-Power Test of a TE11-Mode X-Band RF Window With Taper Transitions," Proc. Particle Accelerator Conference, Dallas, TX 1995, pp. 1590 1592.

[11] O S Lamba et al., "Design and Optimization of Asymmetric RF Window for 6 MW Pulse Power S-band Klystron," Proc. International Conference on Recent Advances in Microwave Theory and Applications, Jaipur, India, 2008, pp. 44 - 46.

[12] George B. Collins, Microwave Magnetrons, , New York, MIT Radiation Laboratory Series, McGraw Hill, 1948, pp. 11,24-37,780.

[13] J. Benford, J. A. Swegle and E. Schamiloglu, "Relativistic Magnetrons and MILOS" in High Power Microwaves, $2^{\text {nd }}$ ed., Boca Raton, FL, USA, Taylor \& Francis, 2007, pp.167- 259-308.

[14] S. Y. Liao, "Microwave Crossed-Field Tubes (M Type)" in Microwave Devices and Circuits, $3^{\text {rd }}$ ed., Englewood Cliffs, NJ, USA, Prentice Hall, 1996, pp. 427-449.

[15] D. Andrey and K. J. Hendricks, "Particle-in-Cell (PIC) Simulation of CW Industrial Heating Magnetron," Journal of Microwave Power and Electromagnetic Energy, 44 (2), 2010, pp. 114-124

[16] X. Chen, M. Esterson and P.A. Lindsay, "3D simulation of microwave Magnetrons ," Proc. IEE Colloquium on High Frequency Simulation: Part Two, London, UK, 1997, pp.1-4.

[17] A. Leggieri, D. Passi and F. Di Paolo, "Multiphysics Modeling Based Design of a Key-Holes Magnetron," Proc. of IEEE International Conference on Numerical Electromagnetic Modeling and Optimization, Pavia, Italia, 2014, in press.

[18] COMSOL, COMSOL Multiphysics User's Guide, Version 4.3, Stockholm, SW, COMSOL AB., 2012, pp. 48-938.

[19] A. Leggieri, A. Ciccotelli, G. Felici, L. Zappelli, D. Passi and F. Di Paolo, "Tuned Window for Standing Wave Linear Accelerators," Proc. Progress in Electromagnetic Research Symposium, Guangzhou, China, 2014.

[20] A. C. Ugural, "Stress and Strain," in Mechanical Design: An Integrated Approach, $1^{\text {st }}$ ed., New York, McGraw-Hill, 2003, pp. 7273.

[21] J. R. Pierce, "Simple Electron Motion" in "Theory and Design of Electron Beams", The Bell Telephone Laboratories Series, New York, D. Van Nostrand Company, 1954, pp. 26-28.

[22] F. Caspers "RF engineering basic concepts: the Smith chart", CERN, Geneva, pp.14-16.

[23] Samuel Liao "Microwave devices and circuits", Prentice Hall, Englewood Cliffs, New Jersey, pp.432-433.

[24] F. Di Paolo, "Fundamental Theory of Transmission Lines," in "Networks and Devices Using Planar Transmission Lines", $1^{\text {st }}$ ed., Boca Raton, FL, CRC Press LLC, 2000, pp. 19-81.

[25] N. Marcuvitz, Sec. "Capacitive Obstacles of Finite Thickness" of Chap. "Four Terminal Structures," in "Waveguide Handbook", $1^{\text {st }}$ ed., New York, McGraw-Hill, 1951, pp.248-257.

[26] A. Leggieri, F. Di Paolo, D. Passi, A. Ciccotelli and G. Felici, "High Transparent Matched Window for Standing Wave Linear Accelerators," Proc. of Linear Accelerator Conference LINAC, Geneva, 2014. 